\title{
Interaction of a Polymer of Intrinsic Microporosity (PIM-1) with Penetrants
}

\author{
Nhamo Chaukura ${ }^{1,}$, , Louise Maynard-Atem² \\ ${ }^{1}$ Polymer Science \& Engineering Department, Harare Institute of Technology, Harare, Zimbabwe \\ ${ }^{2}$ School of Chemistry, University of Manchester, Manchester, UK
}

Email address:

LMaynardAtem@live.co.uk (L. Maynard-Atem),nchaukura@gmail.com (N. Chaukura)

\section{To cite this article:}

Nhamo Chaukura, Louise Maynard-Atem. Interaction of a Polymer of Intrinsic Microporosity (PIM-1) with Penetrants. American Journal of Applied Chemistry. Vol. 3, No. 3, 2015, pp. 139-146. doi: 10.11648/j.ajac.20150303.17

\begin{abstract}
The characterisation, solubility testing, and alcohol adsorption studies of PIM-1 are reported. Techniques used for characterisation are $\mathrm{N}_{2}$ sorption and ATR-FTIR analysis. A number of solvents were tested for their ability to dissolve low molecular weight $\left(\sim 30000 \mathrm{gmol}^{-1}\right)$, medium molecular weight $\left(\sim 60000 \mathrm{gmol}^{-1}\right)$, and high molecular weight $\left(\sim 120000 \mathrm{gmol}^{-1}\right)$ PIM-1.Results showed solubility of PIM-1 in these solvents to be dependent on molar mass. $\mathrm{CHCl}_{3}$, tetrahydrofuran (THF), $\mathrm{CH}_{2} \mathrm{Cl}_{2}$, dicholobenzene (DCB), 1,2,4- trichlorobenzene (TCB), and acetophenone were found to be good solvents at all molecular weights. Treatment of PIM-1 with nonsolvents, namely: small alcohols and water, followed by solvent removal, caused swelling, resulting in an increase in BET surface area of about $11 \%$, except for water, which reduced BET surface area by the same amount $(11 \%)$. Methanol $(\mathrm{MeOH})$ treatment resulted in a new population of micropores around $0.6 \mathrm{~nm}$. The change after ethanol $(\mathrm{EtOH})$ and propanol $(\mathrm{PrOH})$ treatment was not as significant. Hydrogen bonding between alcohols and PIM-1 was also investigated using shifts in the frequencies of FTIR peaks for the ether and the nitrile functional groups. Nitrile group frequencies for the alcohols were consistently blue-shifted, whereas the ether frequencies were red shifted. Both red- and blue-shifts were used as a measure of hydrogen bond strength.
\end{abstract}

Keywords: PIM-1, Solvents, Alcohols, Hydrogen-Bonding

\section{Introduction}

Possible applications for polymers of intrinsic microporosity (PIMs), specifically PIM-1 (Fig. 1), include hydrogen storage[1], heterogeneous catalysts[2] and gas separation membranes[3] among others. These applications involve PIM-1 interacting with gases or liquids. It therefore becomes important to identify interaction sites and investigate their form.

While some properties can be measured experimentally, others can also be predicted using computational modeling and thus serve as an excellent measure for the accuracy of such molecular simulations. Atomistic modelling is a powerful tool that can be used in both a supportive capacity, to provide explanations for experimentally observed interactions, as well as in a predictive capacity, to identify trends yet to be observed. Using experimental data available for PIM-1, Heuchel and co-workers [4] undertook an atomistic study of the archetypal polymer exploiting both established and novel molecular modeling techniques. A computational model of PIM-1 was built as a consequence and several properties were investigated, including sorption isotherms, gas transport and free volume.

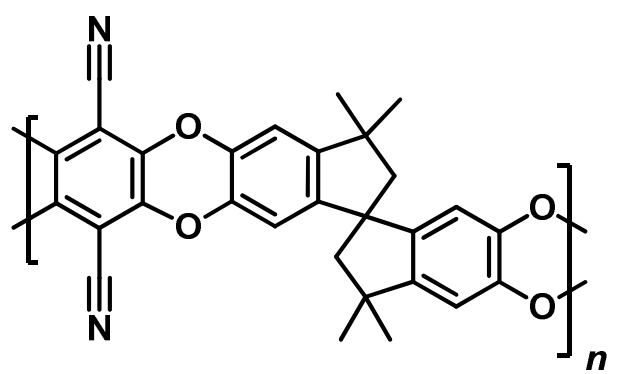

Fig. 1. Molecular structure of PIM-1.

Modelling of PIM-1 membranes gave some valuable insight into the influence the nitrile group has on the permeation properties of the polymer. The results of this 
study highlighted that PIM-1 has large solubility and diffusion coefficients for most gases measured. This trend is likely a result of the presence of the nitrile group which leads to a stronger affinity with penetrant gas molecules as well to as a larger fractional free volume. Despite providing an invaluable insight into the nature of the free volume of PIMs (as well confirming the accuracy of the molecular models described) these works investigate interaction mostly from the perspective of the space in between the polymer chains as opposed to the polymer itself. The aromatic backbone of adjoining rings in PIMs confers rigidity to the polymer structure and this feature prohibits large-scale conformational changes by rotation. Despite the lack of rotation, there is still a level of in-plane flexibility in PIM-1. It is this feature that may be responsible for swelling characteristics of PIM-1 on exposure to small molecules.

Recently, the focus has shifted away from investigating the amount of free volume, and has moved towards looking at intermolecular interactions [5]. Such interactions are important due to the marked effect on PIM-1 after alcohol treatment, especially in terms of permeability properties.

Apart from alcohols affecting the transport properties of a membrane; the casting solvent may also have a part to play $[6,5]$. A PIM-1 film cast from $\mathrm{CHCl}_{3}$, for example, has been known to behave differently to a film of the same material cast from THF. The influence of these interactions has been analysed for various polymers, including polyetherimides [5] and perfluorinated polymers [7], as well as PIM-1 [8] and carboxylated analogues of PIM-1 [9] Dipole-dipole interactions and hydrogen bonding have been identified as possible contributors to changes in transport properties; the latter being especially prevalent in polymers where there are ether connector groups present.

For PIM-1, the effect of residual solvent has been studied both experimentally [5] and computationally [8] In both cases the ether linkage and the nitrile group were identified as likely sites for interaction with residual solvents (encompassing casting solvents, lower alcohols and nonsolvents). This paper reports techniques used to investigate (1) the dependence of PIM-1 solubility on molar mass, (2) the effect that lower alcohols, so called residual solvents and water have on the free volume in PIM-1 and (3) on the interaction sites in the polymer.

\subsection{Polymer Solubility}

PIM-1 has good solution processibility properties. The ability to cast polymeric membranes opens up a commercially promising avenue for possible applications. PIM-1 membrane fabrication has been limited to a select group of solvents including chloroform [10, 11], THF and more recently chlorobenzene [12]. The choice of solvent can have an important effect on the properties of the resulting membrane. It is essential to build up a detailed profile of the organics in which the polymer is both soluble and insoluble.
This can allow the prediction of organic solvents that have not yet been physically tested, and also for the preparation of appropriate solvent mixtures. Developing a relationship between a polymer and a solvent requires a multi-component argument. According to Hansen [13, 14], the solubility parameter $(\delta)$ for a solvent is made up of three (non-equally) contributing components. These are: hydrogen bonding force contribution $\left(\delta_{\mathrm{h}}\right)$, dispersion force contribution $\left(\delta_{\mathrm{d}}\right)$, and polar force contribution $\left(\delta_{\mathrm{p}}\right)$. Thus the solubility parameter can be defined by Equation 1 .

$$
\delta^{2}=\delta_{h}^{2}+\delta_{d}^{2}+\delta_{p}^{2}
$$

This multi-component system solubility parameter means that each solvent can be represented by a fixed point on a ternary plot.

\subsection{H-bonding in PIM-1}

PIM-1 displays two vibrational bands that could potentially be used to monitor its hydrogen bonding state: the $-\mathrm{O}$ - stretch $\left(1250-1350 \mathrm{~cm}^{-1}\right)$, and the $-\mathrm{CN}$ stretch (around $\left.1265 \mathrm{~cm}^{-1}\right)$. Generally, the $\mathrm{H}---\mathrm{N}$ and $\mathrm{H}---\mathrm{O}$ bond lengths are 0.275 and $0.272 \mathrm{~nm}$ respectively [15] Yampolskii et al. [5] carried out FT-IR and membrane permeation experiments to study the sensitivity of PIM-1 and polyetherimide (PEI) membrane properties to the presence of low molecular weight compounds that form hydrogen bonds. They showed that such hydrogen bonding causes conformational changes in the polymer matrix, which in turn has a bearing on the performance properties of the membrane. Residual chloroform from the membrane casting stage, for example, was found to alter the positions and relative intensities of IR bands in the region of absorption of the C-O-C bonds. Removing the residual chloroform consequently changed the IR spectrum. They suggested this could be caused by conformational changes in the $\mathrm{Ph}-\mathrm{O}-\mathrm{Ph}$ connector groups due to the heating and desorption of chloroform [5] They also observed shifts in the $1250-1350 \mathrm{~cm}^{-1}$ band widths after impregnating the films with water and lower alcohols (methanol and ethanol), which they interpreted as conformational rearrangement in the polymer. Aromatic ethers are expected to show strong absorption in that region due to the C-O-C asymmetric stretching vibration [16]. A shoulder around $3340 \mathrm{~cm}^{-1}$ is characteristic of the associates $\mathrm{O}-\mathrm{HO}-\mathrm{R}$. The band due to the $\mathrm{OH}$ unperturbed by hydrogen bonding is expected in the region $3400-3600 \mathrm{~cm}^{-1}$, but they observed a marked difference between the $\mathrm{OH}$ band from ethanol $\left(3670 \mathrm{~cm}^{-1}\right)$ and that from water (wide band at 3560 $\mathrm{cm}^{-1}$ ). Interactions between polymer matrix and penetrant can be hydrogen bonding, weak dipole-dipole moments, or $\mathrm{OH}-\pi$ interactions [17]. Hydrogen bonds between polymer chains and low molecular weight molecules can significantly affect the permeation and sorption parameters [18]. Figure 2 shows the IR peaks due to the $-\mathrm{CN}$ and -O-groups in PIM- 1 . 


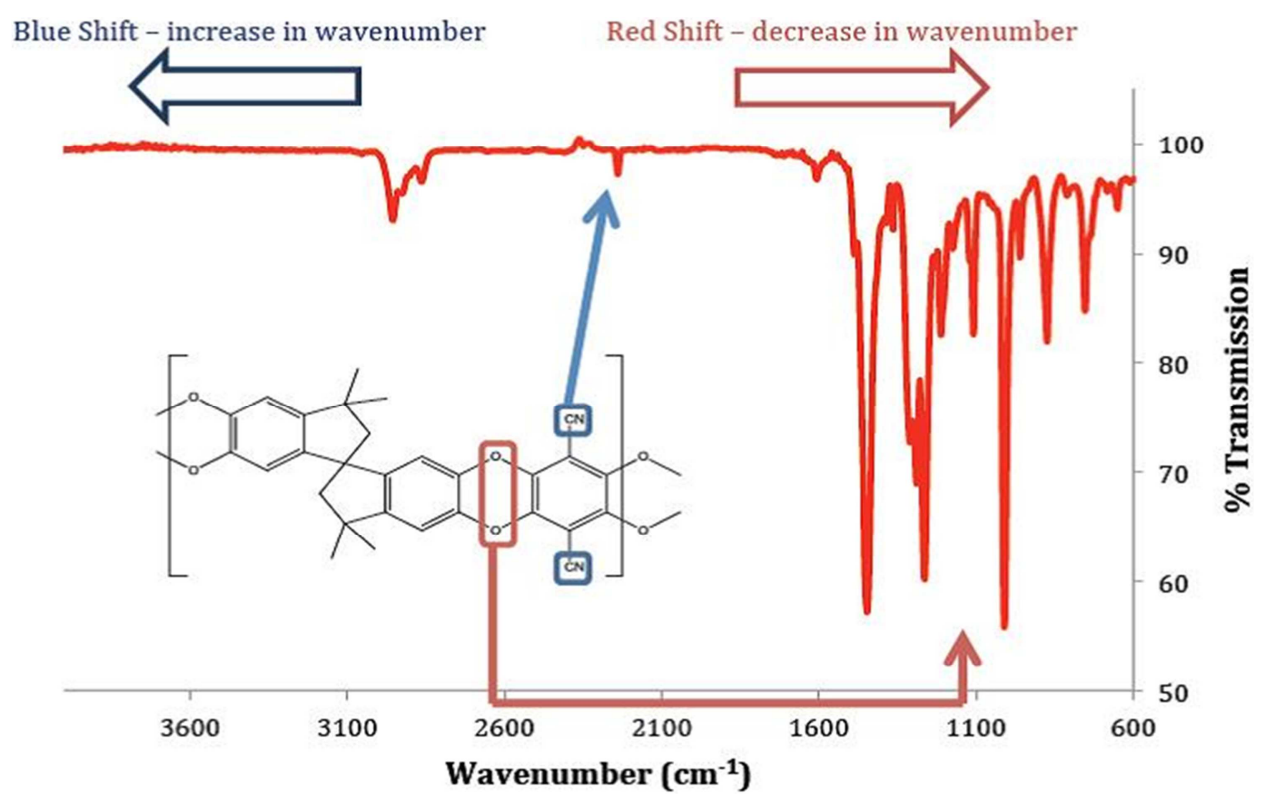

Fig. 2. IR spectrum for PIM-1 with likely interaction sites highlighted (-CN group and ether linkage). Inset-PIM-1 chemical structure. Any shifts in the wavenumber of either peak can be characterized as either a red shift (decrease in wavenumber) or a blue shift (increase in wavenumber) [19].

\section{Materials and Methods}

\subsection{Materials}

Solvents and other chemical reagents were purchased from Sigma-Aldrich. PIM-1 was synthesized according to the method reported by Budd et al. [11].

\subsection{Solubility Testing}

A list of the solvents used in this investigation, is presented in Table 1. Three different PIM-1 samples of molar masses 30000,60000 and 120000 gmol $^{-1}$ determined using the method described by Ghanem et al.[20], were assessed in order to identify any transient solvents (i.e. solvents where low molar mass PIM-1 is soluble, but high molar mass polymer is insoluble). PIM-1 (50 mg) was dissolved by stirring in a solvent $(5 \mathrm{~mL})$ overnight; the resulting solutions were separated into the following categories:

Good Solvents denoted by PIM-1 dissolving completely without excessive agitation, completely homogeneous solution results.

Partial Solvents denoted by majority dissolution of polymer, remainder of material forms a gel.

Slight Solvents denoted by majority of the polymer being completely insoluble and sinking to the bottom of the vial, the solvent becomes coloured indicating an amount of dissolution.

Non solvents denoted by completely insolubility of the polymer, the solvent remains colourless.

\subsection{Characterisation}

The swelling of PIM-1 on exposure to penetrants like alcohols and water were studied using $\mathrm{N}_{2}$ sorption experiments, and H-bonding patterns and sites were investigated using FTIR.
$N_{2}$ sorption $\mathrm{N}_{2}$ sorption studies at $77 \mathrm{~K}$ were undertaken using a Beckman Coulter 3100 Surface Area Analyzer (Fullerton, California, USA). Before sorption analysis, the sample was outgassed under vacuum at a temperature of $120{ }^{\circ} \mathrm{C}$ for a period of $960 \mathrm{~min}$. Helium was used for the free space determination, after sorption analysis, both at ambient temperature and at $77 \mathrm{~K}$. The apparent surface area was calculated from $\mathrm{N}_{2}$ adsorption data by multi-point Brunauer, Emmet, Teller (BET) analysis and pore size distributions were determined using the Horvath-Kawazoe (H-K) method.

FTIR Analysis IR spectra were recorded on a Bio-Rad FTS6000 spectrometer equipped with an ATR sample handling system, and annexed to a Whatman FTIR purge gas generator. The spectra were recorded in the attenuated total reflection (ATR) mode, with a resolution of $0.25 \mathrm{~cm}^{-1}$, a sensitivity of 1 , and 16 scans in the range $4000-600 \mathrm{~cm}^{-1}$ were averaged for each spectrum.

\subsection{Alcohol Treatments}

Freshly prepared PIM-1 samples (100 mg each) were separately soaked in three different alcohols (methanol, ethanol and propanol) and water (approximately $10 \mathrm{~mL}$ ) overnight. The PIM-1 samples were then dried in a vacuum oven at $120{ }^{\circ} \mathrm{C}$ for a period of $24 \mathrm{~h}$. Separate specimens of the samples were then exposed to low pressure $\mathrm{N}_{2}$ sorption and FTIR measurement.

\section{Results and Discussion}

\subsection{Solubility of PIM-1}

Table I presents the solubility behaviour of samples of PIM-1 of high $\left(\sim 120000 \mathrm{~g} \mathrm{~mol}^{-1}\right)$, medium $\left(\sim 60000 \mathrm{~g} \mathrm{~mol}^{-1}\right)$ and low $\left(\sim 30000 \mathrm{~g} \mathrm{~mol}^{-1}\right)$ molar mass. The highest molar mass is in the range that is readily film-forming, while the 
lowest molar mass would not normally give robust membranes.

Table I. Results of the solubility investigation on (a) high molar mass ( $M$ $\left.120000 \mathrm{~g} \mathrm{mo}^{-1}\right)$, (b) medium molar mass $\left(M \sim 60000 \mathrm{~g} \mathrm{mo}^{-1}\right)$, and (c) low molar mass $1\left(M \sim 300000 \mathrm{~g} \mathrm{mo}^{-1}\right)$ PIM-1.

\begin{tabular}{|c|c|c|c|}
\hline Good solvents & $\begin{array}{l}\text { Partial } \\
\text { solvents }\end{array}$ & Slight solvents & Non-solvents \\
\hline \multicolumn{4}{|c|}{ High molar mass PIM-1 (M 120000 gmol-1) } \\
\hline Chloroform & Chlorobenzene & Dichloroethane & Water \\
\hline Dichloromethane & Benzaldehyde & o-Xylene & Methanol \\
\hline Dichlorobenzene & & Acetone & Ethanol \\
\hline $\begin{array}{l}1,2,4- \\
\text { Trichlorobenzene }\end{array}$ & & Toluene & Hexane \\
\hline \multirow[t]{4}{*}{ Acetophenone } & & DMSO & Glycerol \\
\hline & & 1,4-Dioxane & Cyclohexane \\
\hline & & DMF & Butanol \\
\hline & & DMAc & Propan-1-ol \\
\hline \multicolumn{4}{|c|}{ Medium molar mass PIM-1 (M 60 000 gmol-1) } \\
\hline Chloroform & Chlorobenzene & p-Xylene & Water \\
\hline Dichloromethane & o-Xylene & Acetone & Methanol \\
\hline Dichlorobenzene & Dichloroethane & Toluene & Ethanol \\
\hline $\begin{array}{l}1,2,4- \\
\text { Trichlorobenzene }\end{array}$ & & DMSO & Hexane \\
\hline Acetophenone & & 1,4-Dioxane & Glycerol \\
\hline Benzaldehyde & & DMF & Cyclohexane \\
\hline THF & & DMAc & $\begin{array}{l}\text { Butanol } \\
\text { Propan-1-ol }\end{array}$ \\
\hline \multicolumn{4}{|c|}{ Low molar mass PIM-1 (M 30000 gmol-1) } \\
\hline Chloroform & Dichloroethane & Acetone & Methanol \\
\hline THF & p-xylene & Toluene & Ethanol \\
\hline Dichloromethane & o-xylene & DMSO & Hexane \\
\hline Dichlorobenzene & & 1,4-Dioxane & Glycerol \\
\hline $\begin{array}{l}1,2,4- \\
\text { Trichlorobenzene }\end{array}$ & & DMF & Cyclohexane \\
\hline Acetophenone & & DMAc & Butanol \\
\hline Benzaldehyde & & & Propan-1-ol \\
\hline
\end{tabular}

From Table I, it can be established that from the twentyfive solvents tested, there are six solvents in which PIM-1 is soluble at all molecular weights: Chloroform, THF, Dichloromethane, Dichlorobenzene, 1,2,4-Trichlorobenzene and Acetophenone.

Comparing the Hansen solubility parameters [14] for this set of solvents, some similarities become apparent. The proportional representations for each solvent show that the dispersion force has the largest contribution at $\sim 63 \%$. Both the polar force and hydrogen bonding contributions are relatively small, indicating that particularly polar or $\mathrm{H}$ bonding solvents are a poor choice if solubility of PIM-1 is the desired outcome. H-bonding between PIM-1 and water/alcohols is treated in more detail further in this paper.

Molar mass has an effect on the solubility of PIM-1. As $M$ decreases from 'high' to 'medium'; benzaldehyde becomes a good solvent and dichloroethane and $o$-xylene become partial solvents. For the low $M$ sample, other transient solvents can be identified; chlorobenzene becomes a good solvent and p- xylene becomes a partial solvent.

Apparently, the lower the molar mass of the polymer, the more solvents will fall into the 'Good Solvent' category for two reasons. Firstly, there are more polymer chain ends and much less capacity for polymer chain entanglement, and secondly, there is a substantial amount of cyclic material at low molar mass (whether cyclic persist at high molar mass has yet to be determined), which do not contain any endgroup but have been found to be soluble in the reaction solvent used during PIM-1 synthesis [21]. There are no changes in the non-solvent category, which is perhaps not surprising; these solvents are more likely to become transient at very low molar masses, but this hypothesis is not tested here. With the exception of cyclohexane, the dispersion force contributions [14] are all less than $50 \%$, which is the opposite of the trend seen for 'Good Solvents'.

\subsection{Swelling of PIM-1 in Lower Alcohols}

$\mathrm{N}_{2}$ Sorption and Pore Size Distribution Investigation $\mathrm{N}_{2}$ sorption was used to analyse how PIM-1 interacts with small organic molecules because it produces results that are easily compared, both visually and numerically. The isotherms (Fig.3) and BET surface areas (Table II) were compared. There is a marked increase in the BET surface area ( $\sim 11 \%)$ and a change in the effective micropore distribution after the polymer has been exposed to alcohols, though this increase lessens as the size of the alcohol increases. A decrease in BET surface area $(\sim 11 \%)$ is seen when PIM-1 is exposed to water for a prolonged period of time. It is important to note that traces of water can interact very strongly with the polymer and occupy the free volume within the structure in both solid and membrane form, therefore it is perhaps not surprising that some of the free volume becomes inaccessible after exposure to water. To maximize consistency in the work discussed, each solvent treatment was carried out on freshly precipitated PIM-1 samples to minimize the effect of sample history on the results that follow.

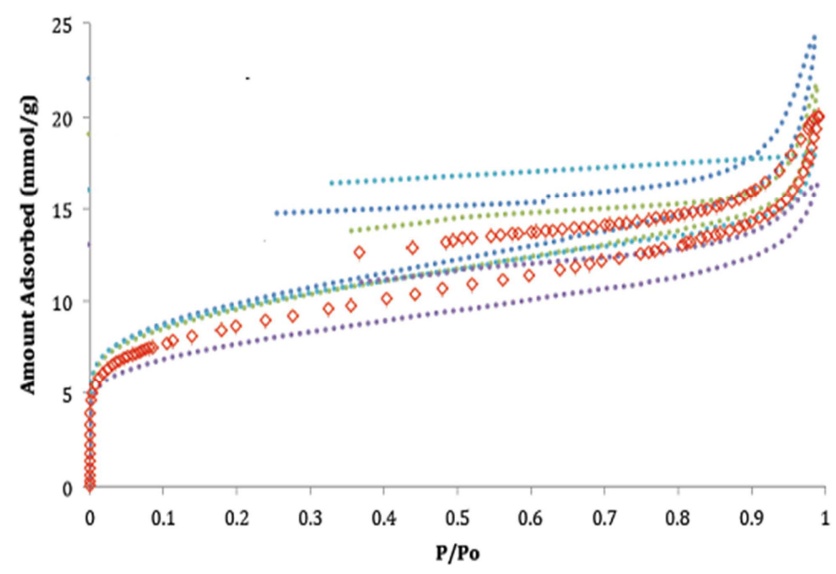

Fig. 3. $\mathrm{N}_{2}$ sorption isotherms for PIM-1 samples that have undergone various solvent. PIM-1 with no treatment; red diamonds, PIM-1 after $\mathrm{MeOH}$ treatment; dark blue dots, PIM-1 after EtOH treatment; green dots, PIM-1 after $\mathrm{PrOH}$ treatment; light blue dots, and PIM-1 after $\mathrm{H}_{2} \mathrm{O}$ treatment; purple dots. 
Table II. Average BET surface areas (S $\left.S_{B E T}\right)$ for PIM-1 samples that underwent various alcohol treatments.

\begin{tabular}{llll}
\hline BET Surface Area $\left(\mathbf{S}_{\mathbf{B E T}}\right)\left( \pm \mathbf{5} \mathbf{~ m}^{\mathbf{2}} / \mathbf{g}\right)$ & & \\
\hline Swelling agent & Before swelling & After swelling & $\boldsymbol{\Delta S}_{\text {BET }}$ \\
\hline None & 685 & - & - \\
$\mathrm{MeOH}$ & 788 & 891 & +103 \\
$\mathrm{EtOH}$ & 759 & 833 & +74 \\
$\mathrm{PrOH}$ & 770 & 855 & +85 \\
$\mathrm{H}_{2} \mathrm{O}$ & 614 & 543 & -71 \\
\hline
\end{tabular}

The pore size distributions for PIM-1 without treatment and $\mathrm{MeOH}, \mathrm{EtOH}, \mathrm{PrOH}$, and $\mathrm{H}_{2} \mathrm{O}$ treated PIM-1 are

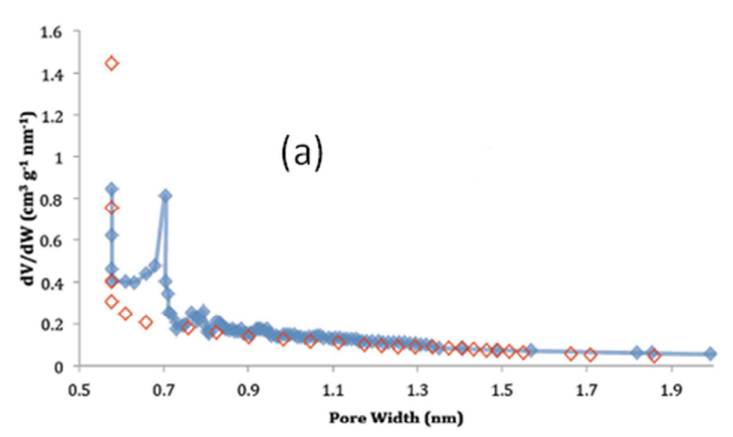

compared in Fig. 4 (a), (b), (c) and (d) respectively. The peak at approximately $0.6 \mathrm{~nm}$ is characteristic of all PIM-1 samples. This is not a real peak, but an artefact of the methodology [22]. However a novel peak at about $0.7 \mathrm{~nm}$ infers that the methanol treatment might have opened up previously unavailable porosity. This newly available population of peaks around $0.7 \mathrm{~nm}$ may correspond to the removal of previously adsorbed solvents which were clogging the free volume within the material. The new peak may also be a result of the polymers ability to swell and adsorb more probe molecules. Both these possible explanations are consistent with the increase in BET surface area seen for the $\mathrm{MeOH}$ treated sample.
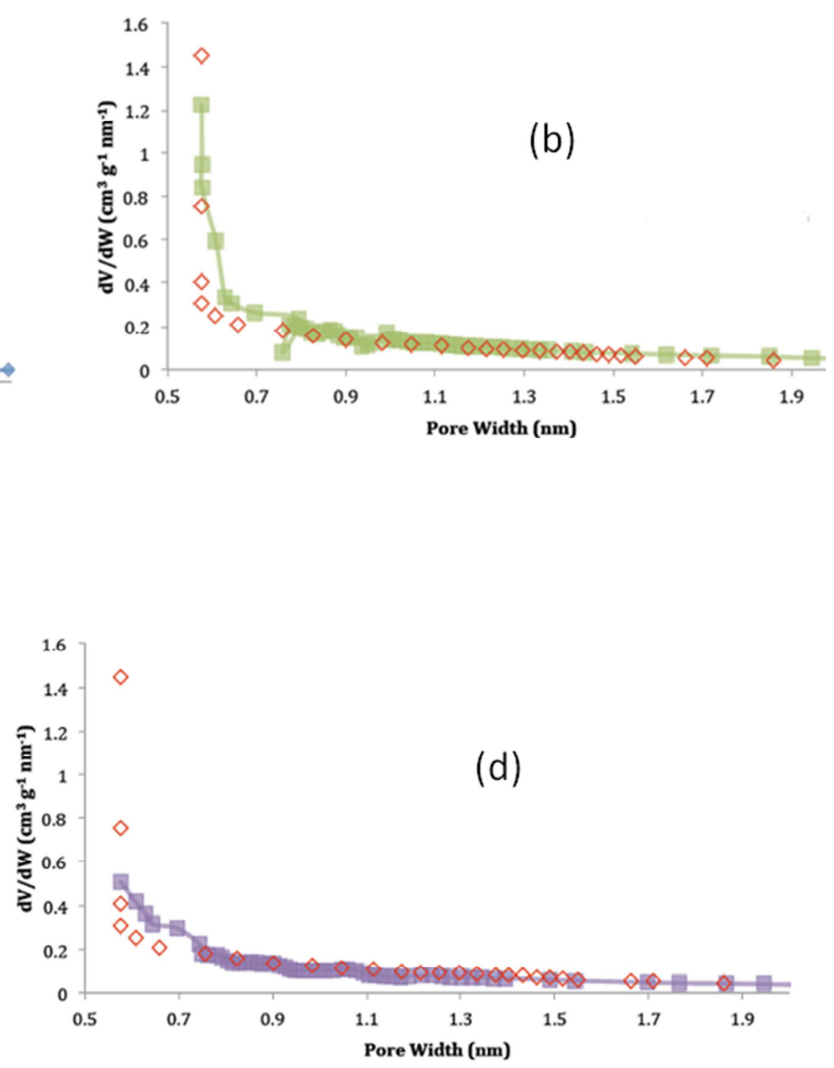

Fig. 4. H-K pore size distributions for (a) PIM-1 (red diamonds) and MeOH treated PIM-1 (blue diamonds), (b) PIM-1 (red diamonds) and EtOH treated PIM1 (green squares), (c) PIM-1 (red diamonds) and PrOH treated PIM-1 (blue squares), and (d) PIM-1 (red diamonds) and $\mathrm{H}_{2} \mathrm{O}$ treated PIM-1 (purple squares).

The pore size distributions for PIM-1 without treatment and EtOH exposed PIM-1 are compared in Fig. 4 (b). The two distributions are similar in shape and there are no novel peaks (as was the case with $\mathrm{MeOH}$ treated PIM-1). This may infer that the increase in BET surface observed is not related to previously inaccessible porosity and is a consequence of the removal of residual contaminants in the polymer.

The pore size distributions for PIM-1 without treatment and Pr-OH exposed PIM-1 are compared in Fig. 4 (c). The main peak for this data is slightly shifted to the right, but is still very close to $0.6 \mathrm{~nm}$, thus it can be assumed that the arrangement of pores within both samples has not been significantly altered by the alcohol treatment. The shape of the distribution is generally the same as non-treated PIM-1, if slightly noisier; again the increase in surface area may be attributable to the removal of residual contaminants in the sample.

The pore size distributions for PIM-1 without treatment and $\mathrm{H}_{2} \mathrm{O}$ exposed PIM-1 are compared in Fig. 4 (d). It seems, given how low the main peak of the data falls, that a large proportion of the pore volume has been lost. This observation is consistent with the loss of accessible porosity, and the reduced BET surface that was observed for this sample; it is also consistent with the fact that water can become very tightly bound and hence persistent within the PIM-1 structure (due to hydrogen bonding) and fill some of the polymer's free volume.

Exposing PIM-1 powder samples to alcohol reveals a relationship between the size of the alcohol molecules and the change in apparent free volume (as measured by BET 
surface area and $\mathrm{H}-\mathrm{K}$ pore size distributions). $\mathrm{MeOH}$, the smallest member of the alcohol series, shows the largest increase in BET surface area as well as the greatest structural differences according to the pore size distributions, whereas $\mathrm{PrOH}$ (the largest member of the series analysed) appears to have the least effect on the capacity of the polymer. Given that PIM-1 is a microporous polymer, the trend observed for the alcohols used is as expected, as the smaller molecules are more likely to have enhanced access to the pores within the structure. The pronounced effect of $\mathrm{MeOH}$ on PIM-1 samples is evident in terms of permeability [18] and agrees with the results discussed here. It is important to discuss whether or not the changes described in this section are greater than experimental error, which are inherent in the methodology and cannot be easily or entirely removed.

While the $\mathrm{N}_{2}$ sorption measurements described above were carried out on powder PIM-1 samples; it is possible to use membranes in such analyses but the data is much more difficult to obtain and much less reliable from the point of view of an $\mathrm{N}_{2}$ probe molecule used in this technique.

\subsection{Hydrogen Bonding}

Figure 5 shows an interesting pattern on the variation of the frequency shift with alcohol chain length. It appears that, as the alcohol chain length increases, so does the frequency shift up to ethanol, after which the frequency shift decreases. Perhaps this could be attributed to steric hindrance of the bulkier groups so that their access to the hydrogen bonding site is progressively restricted. Thereafter the frequency shift decreases with increasing chain length. The biggest difference in frequency shifts is between water and the alcohols. Alcohols exhibit anomalous frequency shifts (blue shifts) on the nitrile group. There is a contestable hypothesis that this could be due to intramolecular nitrile-nitrile interactions rather than hydrogen bonding. There may be such interactions in the unswollen state, so that the baseline value is red-shifted from the value that would be expected for a free nitrile. Swelling would move chains, and hence nitrile groups, further apart, leading to an apparent blue shift. Blue shifts have been observed by Wojtkow et al. as they investigated the effect of temperature on butyl-alcohol mixtures [23]. On performing IR spectroscopy on triformylmethane (TFM), Lord et al. also observed a blue shift of the C-H stretch [24]. These researchers however, did not explain this unusual phenomenon. An explanation of the blue shift was proposed by Hobza et al. who ascribed the descriptive nomenclature improper blue shifting hydrogen bond as opposed to the normal red shifting bond [19]. They observed that the blue shifting hydrogen bond is accompanied by the shortening of the O-H bond.

There are no fundamental differences between red and blue shifting hydrogen bonds [25]. Joseph and Jemmims demonstrated that the D-H---A energy minimum can occur at a longer, equal or shorter D-H distance relative to the D-H fundamental [26]. For the red shifting hydrogen bond, elongation of the $\mathrm{O}-\mathrm{H}$ bond enhances the dipole-dipole attraction between the alcohol and proton acceptor, $\mathrm{O}$ or $\mathrm{N}$.
The electrostatic interaction is predominant and other terms like the charge transfer term are also significant. In contrast however, in improper blue shifting hydrogen bonds, the predominant stabilisation energy contribution is from the London forces term, which varies inversely with the sixth power of the separation between the centres of mass of the proton donor/acceptor system [27]. To maximise this attraction, the $\mathrm{O}-\mathrm{H}$ bond length shortens. Boldeskul et al. explain this occurrence in chloroform-nitro systems as an increase in the $\mathrm{s}$ character of the $\mathrm{C}-\mathrm{H}$ bond owing to molecular deformation caused by intermolecular forces [28]. Admittedly, this does not account for all systems involving improper blue shift hydrogen bonding, so additional terms are required to adequately explain this type of hydrogen bonding. In redefining the hydrogen bond, the IUPAC included the red shift as a criterion for hydrogen bonding, with a caveat as a footnote [17].

Notwithstanding the foregoing possibility, that the nitrile group frequencies for alcohols are consistently blue shifted, suggests a different kind of interaction between the alcohols and these two functional groups. Perhaps this could be ascribed to the sterical orientation of these moieties on the PIM backbone. The structure of PIM-1 (included in Fig. 2) is such that the nitrile nitrogen atoms are more exposed than the ether linkages. This might mean the nitrile groups are more likely to interact with each other in the swollen state of the polymer, and the adsorption of alcohols undermines this interaction.

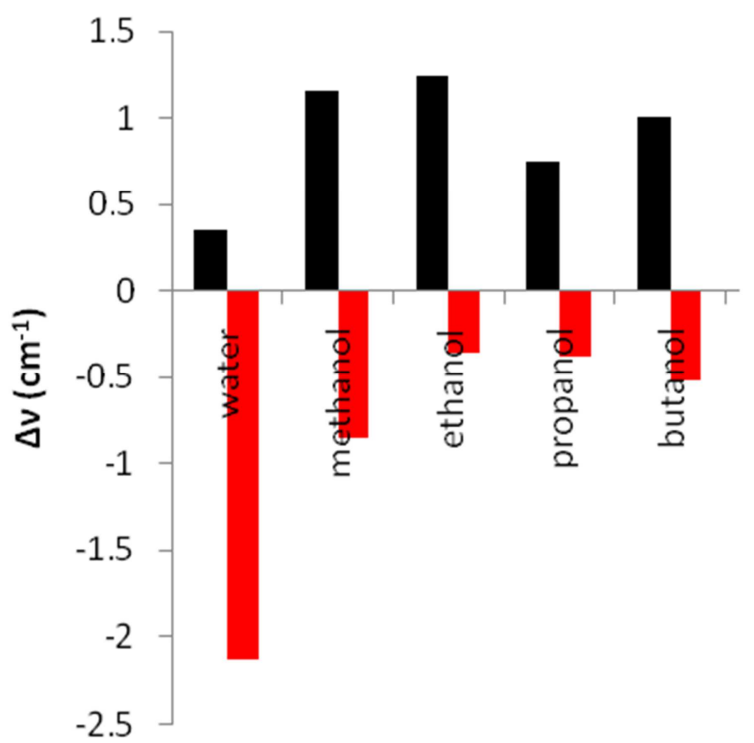

Fig. 5. Frequency shifts $(\Delta v)$ of the $(D H-X)$ band for different hydrogen bonding agents. The black bars represent the nitrile frequency shifts, and the red bars represent the aromatic ether frequency shift. Water and alcohols show a red shift for the aromatic ether group, and a blue shift for the nitrile group.

Because the red shift is correlated to the hydrogen bond strength [19, 28, 29], relative magnitudes of frequency shifts have been used to compare hydrogen bond strengths. The larger the red shift, the stronger the hydrogen bonding causing it. This is because the vibration of a strongly 
hydrogen bonded atom will be more retarded than a weakly bonded one, resulting in a larger red shift. This being the case, the strength of the hydrogen bonds follows the order: water $>$ methanol $>$ butanol $>$ propanol $>$ ethanol with respect to aromatic ether $\Delta v$. Corollary to this, assuming the nitrile groups are also involved in hydrogen bonding, the larger the blue shift the stronger the hydrogen bonding. It follows then that the hydrogen bond strength is in the order: ethanol $>$ methanol $>$ butanol $>$ propanol $>$ water, with respect to the nitrile $\Delta v$. Pandurangi et al. [30] used the difference in absorbance of the aromatic ether stretch, and the change in intensity, $\Delta v$ of the hydroxyl band in order to measure relative hydrogen bond strengths. They demonstrated that this is as good a comparator of bond strength as $\Delta v$.

The formation of a hydrogen bond is accompanied by a weakening and elongation of the ether linkage, thus decreasing the functional group's stretching frequency; this is entirely commonplace where hydrogen bonding agents (such as alcohols) are involved. An increase in intensity as well as a broadening of the peak is also seen for the ether linkage in the presence of alcohols, which lends further evidence to the case for conventional hydrogen bonding. The fact that the strength of this hydrogen bonding appears to decrease as the size of the alcohol increases, points to steric hindrance of the bulkier molecules restricting access to the ether oxygen atoms (Fig. 6).

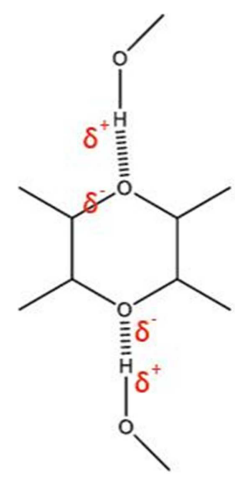

Fig. 6. Hydrogen bonding at the ether linkage in PIM-1.

\section{Conclusion}

\subsection{Solubility}

PIM-1 solubility is molecular weight dependent. Low molecular weight polymer $\left(\sim 30 \quad 000 \mathrm{gmol}^{-1}\right)$ dissolved in Chloroform, THF, Dichloromethane, Dichlorobenzene, 1,2,4Trichlorobenzene, Acetophenone, Benzaldehyde; medium molecular weight polymer $\left(\sim 60000 \mathrm{gmol}^{-1}\right)$ dissolved in Chloroform, Dichloromethane, Dichlorobenzene, 1,2,4Trichlorobenzene, Acetophenone, Benzaldehyde, THF; and high molecular weight polymer $\left(\sim 120000 \mathrm{gmol}^{-1}\right)$ dissolved in $\mathrm{CHCl}_{3}, \mathrm{CH}_{2} \mathrm{Cl}_{2}, \mathrm{DCB}, \mathrm{TCB}$, Acetophenone. The solvents $\mathrm{CHCl}_{3}, \mathrm{THF}, \mathrm{CH}_{2} \mathrm{Cl}_{2}, \mathrm{DCB}, \mathrm{TCB}$, and acetophenone were found to be good solvents at all molecular weights. These are therefore solvents that can be used to process PIM-1 into films and coatings for various applications.

\subsection{Swelling of PIM-1}

$N_{2}$ sorption Treatment of PIM-1 with small alcohols and water, followed by solvent removal, caused swelling, which resulted in an increase in BET surface area of about $11 \%$, except for water, which reduced BET surface area by the same amount $(11 \%)$. MeOH treatment resulted in a new population of micropores around $0.6 \mathrm{~nm}$. The change due after EtOH and PrOH treatment was not as significant.

H-bonding The nitriles exhibit blue shifting in the presence of alcohols may be concordant with the idea of the functional groups becoming less interacting and moving towards the free nitrile position. Alternatively, the blue shifts observed may be attributed to the so-called 'improper blue shifting hydrogen bond' $[19,25,31]$ phenomenon. This type of bonding differs from a regular red shifting hydrogen bond in the way that it is associated with bond contraction instead of a bond elongation. Dipole-dipole interactions between the proton donor and proton acceptor are the main driving forces behind the conventional red-shifting hydrogen bond, whereas it is proposed that electrostatic interactions come to the fore with respect to the blue shifting hydrogen bond [19]. A recent example of blue-shifting hydrogen bonds in relation to nitrile groups has attributed the occurrence of this unusual type of bonding to electrostatic changes in the nitrile environment upon introduction of the proton donor [32].

\section{Acknowledgements}

The authors are grateful to the Engineering and Physical Sciences Research Council, UK (EPSRC Grants EP/D074460/1 and EP/GO65144/1) for providing funding for this research.

\section{References}

[1] N.B. McKeown, P.M. Budd, and D. Book "Microporous Polymers as Potential Hydrogen Storage Materials," Macromol. Rapid Commun., vol. 28, pp 995-1002, 2007.

[2] H. J. Mackintosh, P.M. Budd, and N.B. McKeown, "Catalysis by microporous Phthalocyanine and porphyrin network polymers," J. Mater. Chem., vol. 18, pp 573-578, 2008.

[3] P.M. Budd, K.J. Msayib, C.E. Tattershall, B.S. Ghanem, K.J. Reynolds, N.B. McKeown, and D. Fritsch, "Gas separation membranes from polymers of intrinsic microporosity," J. Membr. Sci., vol. 251, pp. 263-269, 2005.

[4] M. Heuchel, D. Fritsch, P.M. Budd, N.B. McKeown, and D. Hofmann, "Atomistic packing model and free volume distribution of a polymer with intrinsic microporosity (PIM1)," J. Membr. Sci., vol. 318, pp. 84-89, 2008.

[5] Y. Yampolskii, A. Alentiev, G. Bondarenko, Y. Kostina, and M. Heuchel, "Intermolecular Interactions: New Way to Govern Transport Properties of Membrane Materials," Ind. Eng. Chem. Res., vol. 49, pp. 12031-12037, 2010.

[6] G. Larsen, P. Lin, F. Siperstein, and C. Colina, "Methane adsorption in PIM-1," Adsorption, vol. 17, pp. 21-26, 2010. 
[7] J.C. Jansen, M. Macchione, and E. Drioli, "On the unusual solvent retention and the effect on the gas transport in perfluorinated Hyflon ADR membranes," J. Membr. Sci., vol. 287, pp. 132-137, 2007.

[8] L. Zhang, W. Fang, and J. Jiang, "Effects of Residual Solvent on Membrane Structure and Gas Permeation in a Polymer of Intrinsic Microporosity: Insight from Atomistic Simulation," J. Phys. Chem. C, vol. 115, pp. 11233-11239, 2011.

[9] J. Weber, N. Du, and M.D. Guiver, "Influence of Intermolecular Interactions on the Observable Porosity in Intrinsically Microporous Polymers," Macromolecules, vol. 44, pp. 1763-1767, 2011.

[10] P.M. Budd, "Solution-processed, organophilic membrane derived from a polymer of intrinsic microporosity," Adv. Mater., vol. 16, pp. 456, 2004.

[11] P.M. Budd, B.S. Ghanem, S. Makhseed, N.B. McKeown, K.J. Msayib, and C.E. Tattershal, "Polymers of intrinsic microporosity (PIMs): robust, solution-processable, organic nanoporous materials," Chem. Commun. pp. 230-231, 2004.

[12] N.A. Rakow, M.S. Wendland, J.E. Trend, R.J. Poirier, D.M. Paolucci, S.P. Maki, C.S. Lyons, and M.J. Swierczek, "Visual Indicator for Trace Organic Volatiles," Langmuir, vol. 26, pp. 3767-3770, 2010

[13] C.M. Hansen, The Three Dimensional Solubility Parameter \& Solvent Diffusion Coefficient - Their Importance In Surface Coating Formulation. Instituttet for Kemi-industri. Technical University of Denmark, 1967.

[14] C. M. Hansen, "The Universality of the Solubility Parameter," Product R\&D, vol. 8, pp. 2-11, 1969.

[15] N.B. McKeown, and P.M. Budd PM, "Exploitation of Intrinsic Microporosity in Polymer-Based Materials," Macromolecules, vol. 43 , pp. 5163-5176, 2010 .

[16] G. Socrates, Infared and Raman characteristic group frequencies, Tables and Charts. 3 edn. John Wiley and Sons. New York, 2001.

[17] E. Arunan, G.R. Desiraju, R.A. Klein, J. Sadlej, S. Scheiner, I. Alkorta, D.C. Clary, R.H. Crabtree, J.J. Dannenberg, P. Hobza, H.G. Kjaergaard, A.C. Legon, B. Mennucci, D.J. Nesbitt "Defining The hydrogen Bond: An account," Pure Appl. Chem., vol. 83, pp 1619-1636, 2011.

[18] P.M. Budd, N.B. McKeown, B.S. Ghanem, K.J. Msayib, D. Fritsch, L. Starannikova, N. Belov, O. Sanfirova, Y. Yampolskii, and V. Shantarovich, "Gas permeation parameters and other physicochemical properties of a polymer of intrinsic microporosity: Polybenzodioxane PIM-1,". J. Membr. Sci., vol. 325, pp 851-860, 2008.

[19] P. Hobza, and Z.K. Havlas, "Blue-Shifting Hydrogen Bonds," Chem. Rev., vol. 100, pp 4253-4264, 2000.

[20] B.S. Ghanem, N.B. Mckeown, P.M. Budd, N.M. Al-Harbi, D. Fritsch, K. Heinrich, L. Starannikova, A. Tokarev, and Y.
Yampolskii, "Synthesis, Characterization, and Gas Permeation Properties of a Novel Group of Polymers with Intrinsic Microporosity: PIM-Polyimides," Macromolecules, vol. 42, pp. 7881-7888, 2009.

[21] J. Song, N. Du, Y. Dai, G.P. Robertson, M.D. Guiver, S. Thomas, and I Pinnau, "Linear High Molecular Weight Ladder Polymers by Optimized Polycondensation of Tetrahydroxytetramethylspirobisindane and 1,4Dicyanotetrafluorobenzene," Macromolecules, vol. 41, pp. 7411-7417, 2008.

[22] N. Chaukura, "Sorption of Gases and Liquids by Polymers of Intrinsic Microporosity (PIMs)," University of Manchester, Manchester, 2011.

[23] D. Wojtków, and M.A. Czarnecki MA, "Two-Dimensional Attenuated Total Reflection Infrared and Near-Infrared Correlation Study of the Structure of Butyl Alcohol/Water Mixtures," Appl. Spectrosc., vol. 61, pp. 928-934, 2007.

[24] R.C. Lord, and F.A. Miller, "Factors Influencing Characteristic Vibrational Frequencies of Molecules: Intramolecular Effects," Appl Spectrosc 10:115-123, 1956.

[25] B.J. van der Veken, W.A. Herrebout, R. Szostak, D.N. Shchepkin, Z. Havlas, and P. Hobza P, "The Nature of Improper, Blue-Shifting Hydrogen Bonding Verified Experimentally," J. Am. Chem. Soc., vol. 123, pp. 1229012293, 2001.

[26] J. Jorly, and D.J. Eluvathingal DJ (2007) "Red-, Blue-, or NoShift in Hydrogen Bonds: A unified Explanation," J. Am. Chem. Soc., vol. 129, pp. 4620-4632, 2007.

[27] A.J. Barnes, "Blue-shifting hydrogen bonds - are they improper or proper?," J. Mol. Struct., vol. 704, pp. 3-9, 2004.

[28] 28.I.E. Boldeskul, I.F. Tsymbal, E.V. Ryltsev, Z. Latajka, and A.J. Barnes, "Reversal of the usual v(C-H/D) spectral shift of haloforms in some hydrogen-bonded complexes," J. Mol. Struct., vol. 436-437, pp. 167-171, 1997.

[29] M. Rozenberg, G. Shoham, I. Reva, and R. Fausto, "Low temperature FTIR spectroscopy and hydrogen bonding in cytosine polycrystals," Spectrochim. Acta A., vol. 60, pp. 463470, 2004.

[30] R.S. Pandurangi, and M.S. Seehra, "Interaction of Silanol Species on Silica with Hydrogen Bonding Agents Studied by Photoacoustic Spectroscopy," Appl. Spectrosc., vol. 45, pp. 673-676, 1991.

[31] P. Hobza, and Z. Havlas, "Improper, blue-shifting hydrogen bond. Theoretical Chemistry Accounts: Theory, Computation, and Modeling," Theor. Chim. Acta, vol. 108, pp. 325-334, 2002.

[32] H. Zou, J. Liu, and J.K. Blasie, "Mechanism of Interaction between the General Anesthetic Halothane and a Model Ion Channel Protein, III: Molecular Dynamics Simulation Incorporating a Cyanophenylalanine Spectroscopic Probe," Biophys. J., vol. 96, pp 4188-4199, 2009. 